\title{
On Reductions and Real Hamiltonian Forms of Affine Toda Field Theories
}

\author{
Vladimir S GERDJIKOV and Georgi G GRAHOVSKI \\ Institute for Nuclear Research and Nuclear Energy, Bulgarian Academy of Sciences, 72 \\ Tsarigradsko chaussee, 1784 Sofia, Bulgaria \\ E-mail: gerjikov@inrne.bas.bg \\ E-mail: grah@inrne.bas.bg
}

This article is a part of the special issue titled "Symmetries and Integrability of Difference Equations (SIDE VI)"

\begin{abstract}
A family of real Hamiltonian forms (RHF) for the special class of affine $1+1$ dimensional Toda field theories is constructed. Thus the method, proposed in [1] for systems with finite number of degrees of freedom is generalized to infinite-dimensional Hamiltonian systems. We show that each of these RHF is related to a special $\mathbb{Z}_{2^{-}}$ symmetry of the system of roots for the relevant Kac-Moody algebra. A number of explicit nontrivial examples of RHF of ATFT are presented.
\end{abstract}

\section{Introduction}

To each simple Lie algebra $\mathfrak{g}$ one can relate a Toda field theory (TFT) in $1+1$ dimensions. It allows the Lax representation:

$$
[L, M]=0
$$

where $L$ and $M$ are first order ordinary differential operators:

$$
\begin{array}{r}
L \psi \equiv\left(i \frac{d}{d x}-i q_{x}(x, t)-\lambda J_{0}\right) \psi(x, t, \lambda)=0, \\
M \psi \equiv\left(i \frac{d}{d t}-\frac{1}{\lambda} I(x, t)\right) \psi(x, t, \lambda)=0 .
\end{array}
$$

whose potentials take values in $\mathfrak{g}$. Here $q(x, t) \in \mathfrak{h}$ - the Cartan subalgebra of $\mathfrak{g}, \boldsymbol{q}(x, t)=$ $\left(q_{1}, \ldots, q_{r}\right)$ is its dual $r$-component vector, $r=\operatorname{rank} \mathfrak{g}$, and

$$
J_{0}=\sum_{\alpha \in \pi} E_{\alpha}, \quad I(x, t)=\sum_{\alpha \in \pi} e^{-(\alpha, \boldsymbol{q}(x, t))} E_{-\alpha} .
$$

By $\pi_{\mathfrak{g}}$ we denote the set of admissible roots of $\mathfrak{g}$, i.e. $\pi_{\mathfrak{g}}=\left\{\alpha_{0}, \alpha_{1}, \ldots, \alpha_{r}\right\}$ where $\alpha_{1}, \ldots, \alpha_{r}$ are the simple roots of $\mathfrak{g}$ and $\alpha_{0}$ is the minimal root of $\mathfrak{g}$. The corresponding TFT is known 
as the affine TFT. The Dynkin graph that corresponds to the set of admissible roots of $\mathfrak{g}$ is called extended Dynkin diagrams (EDD). The equations of motion are of the form:

$$
\frac{\partial^{2} \boldsymbol{q}}{\partial x \partial t}=\sum_{j=0}^{r} n_{j} \alpha_{j} e^{-\left(\alpha_{j}, \boldsymbol{q}(x, t)\right)}
$$

where $n_{j}$ are the minimal positive integers for which $\sum_{j=0}^{r} n_{j} \alpha_{j}=0$.

The operators $L$ and $M$ are invariant with respect to the reduction group $\mathcal{G}_{\mathbb{R}} \simeq \mathbb{D}_{h}$ where $h$ is the Coxeter number of $\mathfrak{g}$. This reduction group is generated by two elements satisfying $g_{1}^{h}=g_{2}^{2}=\left(g_{1} g_{2}\right)^{2}=\mathbb{1}$ which allow realizations both as elements in Aut $\mathfrak{g}$ and in $\operatorname{Conf} \mathbb{C}$. The invariance condition has the form [2]:

$$
C_{k}\left(U\left(x, t, \kappa_{k}(\lambda)\right)\right)=U(x, t, \lambda), \quad C_{k}\left(V\left(x, t, \kappa_{k}(\lambda)\right)\right)=V(x, t, \lambda)
$$

where $U(x, t, \lambda)=-i q_{x}(x, t)-\lambda J_{0}$ and $V(x, t, \lambda)=-\frac{1}{\lambda} I(x, t)$. Here $C_{k}$ are automorphisms of finite order of $\mathfrak{g}$, i.e. $C_{1}^{h}=C_{2}^{2}=\left(C_{1} C_{2}\right)^{2}=\mathbb{1}$ while $\kappa_{k}(\lambda)$ are conformal mappings of the complex $\lambda$-plane. The algebraic constraints (1.6) are automatically compatible with the evolution. A number of nontrivial reductions of nonlinear evolution equations can be found in [3, 4].

Lemma 1. Let $\mathfrak{g}$ be a simple Lie algebra from one of the classical series $\boldsymbol{A}_{r}, \boldsymbol{B}_{r}, \boldsymbol{C}_{r}$ or $\boldsymbol{D}_{r}$ and let $h$ be its Coxeter number and $N_{0}$ - the dimension of the typical representation. Then the characteristic equation of $J_{0}$ taken in the typical representation has the form:

$$
\zeta^{r_{0}}\left(\zeta^{h}-1\right)=0, \quad r_{0}=N_{0}-h .
$$

Remark 1. The constant $r_{0}=0$ for $\mathfrak{g} \simeq \boldsymbol{A}_{r}, \boldsymbol{C}_{r} ; r_{0}=1$ for $\mathfrak{g} \simeq \boldsymbol{B}_{r}$ and $r_{0}=2$ for $\mathfrak{g} \simeq \boldsymbol{D}_{r}$. Solving the inverse scattering problem in the last two cases requires special treatment of the subspaces related to $\zeta=0$.

The present paper is an extension of a conference report of one of us [5]. In Section 2 we outline the spectral properties of the Lax operator. In Section 3 we generalize the theory of RHF proposed in [1, 5] to the ATFT in $1+1$ dimensions. In Section 4 we show how the involution $\tilde{\mathcal{C}}$ of the complexified Hamiltonian dynamics (defined in Section 3 below) can be related to an automorphism $\mathcal{C}^{\#}$ of the corresponding EDD. This allows one to construct the RHF of ATFT related to any Kac-Moody algebra. We present here (a non-exhaustive) list of examples of RHF of ATFT related to several of the classical series of the Kac-Moody algebras. In deriving them we make use of the method proposed in [6] for constructing $\mathbb{Z}_{p}$-symmetries of the EDD; of course our construction needs only involutions $\left(\mathbb{Z}_{2}\right.$-reductions) of EDD.

\section{The spectral properties of $L$}

The reduction conditions (1.6) lead to rather special properties of the operator $L$. Along with $L$ we will use also the equivalent system:

$$
L m(x, t, \lambda) \equiv i \frac{d m}{d x}+i q_{x} m(x, t, \lambda)-\lambda\left[J_{0}, m(x, t, \lambda)\right]=0,
$$


where $m(x, t, \lambda)=\psi(x, t, \lambda) e^{i J_{0} x \lambda}$. Combining the ideas of [7] with the symmetries of the potential (1.6) we can construct a set of $2 h$ fundamental analytic solutions (FAS) $m_{\nu}(x, t, \lambda)$ of (2.1) and prove that:

1. the continuous spectrum $\Sigma$ of $L$ fills up $2 h$ rays $l_{\nu}$ passing through the origin: $\lambda \in$ $l_{\nu}: \arg \lambda=(\nu-1) \pi / h$;

2. $m_{\nu}(x, t, \lambda)$ is a FAS of (2.1) analytic with respect to $\lambda$ in the sector $\Omega_{\nu}:(\nu-1) \pi / h \leq$ $\arg \lambda \leq \nu \pi / h$ satisfying $\lim _{\lambda \rightarrow \infty} m_{\nu}(x, t, \lambda)=\mathbb{1}$

3. to each $l_{\nu}$ one relates a subalgebra $\mathfrak{g}_{\nu} \subset \mathfrak{g}$ such that $\mathfrak{g}_{\nu} \cap \mathfrak{g}_{\mu}=\emptyset$ for $\nu \neq \mu \bmod (h)$ and $\cup_{\nu=1}^{h} \mathfrak{g}_{\nu}=\mathfrak{g}$. The symmetry ensures that each of the subalgebras $\mathfrak{g}_{\nu}$ is a direct sum of $\operatorname{sl}(2)$-subalgebras;

4. on $\Sigma$ the FAS $m_{\nu}(x, t, \lambda)$ satisfy

$$
\begin{array}{r}
m_{\nu}(x, t, \lambda)=m_{\nu-1}(x, t, \lambda) G_{\nu}(x, t, \lambda), \quad \lambda \in l_{\nu}, \\
G_{\nu}(x, t, \lambda)=e^{-i\left(\lambda J_{0} x+f(\lambda)\right) t} G_{0, \nu}(\lambda) e^{i\left(\lambda J_{0} x+f(\lambda)\right) t} \in \mathcal{G}_{\nu},
\end{array}
$$

where $\mathcal{G}_{\nu}$ is the subgroup with Lie algebra $\mathfrak{g}_{\nu}$ and $f(\lambda)$ is determined by the dispersion law of the NLEE: $f(\lambda)=\sum_{k=0}^{r} E_{-\alpha_{k}} / \lambda$;

5. the FAS of (2.1) satisfy:

$$
\bar{C}_{1}\left(m_{\nu}(x, t, \omega \lambda)\right)=m_{\nu-2}(x, t, \lambda), \quad \lambda \in l_{\nu-2},
$$

where $\bar{C}_{1}$ is equivalent to the Coxeter automorphism [8]:

$$
\bar{C}_{1}(X) \equiv C_{1}^{-1} X C_{1}, \quad C_{1}=e^{\frac{2 \pi i}{h} H_{\rho}}, \quad \rho=\frac{1}{2} \sum_{\alpha>0} \alpha ;
$$

obviously $C_{1}^{h}=\mathbb{1}$ and $\bar{C}_{1}\left(J_{0}\right)=\omega^{-1} J_{0}$;

6. the FAS $m_{\nu}(x, t, \lambda)$ satisfy one of the following two involutions:

$$
\bar{C}_{2}\left(m_{\nu}\left(x, t, \lambda^{*}\right)\right)^{\dagger}=C_{2}\left(m_{2 h-\nu+2}^{-1}(x, t, \lambda)\right),
$$

where $C_{2}, C_{2}^{2}=\mathbb{1}$ is conveniently chosen Weyl group element, or

$$
\left(m_{\nu}\left(x, t,-\lambda^{*}\right)\right)^{*}=m_{h-\nu+2}(x, t, \lambda) .
$$

These relations lead to the following constraints for the sewing functions $G_{0, \nu}(\lambda)$ and the minimal set of scattering data:

$$
\begin{aligned}
& \bar{C}_{1}\left(G_{0, \nu}(\omega \lambda)\right)=G_{0, \nu-2}(\lambda), \\
& \bar{C}_{2}\left(G_{0, \nu}^{\dagger}\left(\lambda^{*}\right)\right)=G_{0,2 h-\nu+2}^{-1}(\lambda), \\
& G_{0, \nu}^{*}\left(-\lambda^{*}\right)=G_{0, h-\nu+2}(\lambda) .
\end{aligned}
$$

If $L$ has no discrete eigenvalues the minimal set of scattering data is provided by the coefficients of $G_{0,1}(\lambda), \lambda \in l_{1}$ and $G_{0,2}(\lambda), \lambda \in l_{2}$. All the other sewing functions $G_{0, \nu}(\lambda)$ can be determined from them by applying (2.8), (2.9) or (2.8), (2.10). 


\section{The real Hamiltonian forms of ATFT}

The Lax representations of the ATFT models widely discussed in the literature (see e.g. [2, 9, 10, 6] and the references therein) are related mostly to the normal real form of the Lie algebra $\mathfrak{g}$, see [12]. Our aim here is to:

1. generalize the ATFT to complex-valued fields $\boldsymbol{q}^{\mathbb{C}}=\boldsymbol{q}^{0}+i \boldsymbol{q}^{1}$, and

2. describe the family of RHF of these ATFT models.

We also provide a tool to construct new inequivalent RHF's of the ATFT. This tool is a natural generalization of the one in [1] to $1+1$-dimensional systems. Indeed, the ATFT related to the algebra $s l(n)$ can be written down as an infinite-dimensional Hamiltonian system as follows:

$$
\begin{aligned}
& \frac{d q_{k}}{d t}=\left\{q_{k}, H_{\mathrm{ATFT}}\right\}, \quad \frac{d p_{k}}{d t}=\left\{p_{k}, H_{\mathrm{ATFT}}\right\}, \\
& H_{\mathrm{ATFT}}=\int_{-\infty}^{\infty} d x\left(\frac{1}{2}(\boldsymbol{p}(x, t), \boldsymbol{p}(x, t))+\sum_{k=0}^{r} e^{-\left(\boldsymbol{q}(x, t), \alpha_{k}\right)}\right),
\end{aligned}
$$

where $\boldsymbol{p}=d \boldsymbol{q} / d t$ and $\boldsymbol{q}$ are the canonical momenta and coordinates satisfying canonical Poisson brackets:

$$
\left\{q_{k}(x, t), p_{j}(y, t)\right\}=\delta_{j k} \delta(x-y) .
$$

Next we introduce an involution $\mathcal{C}$ acting on the phase space $\mathcal{M} \equiv\left\{q_{k}(x), p_{k}(x)\right\}_{k=1}^{n}$ as follows:

$$
\begin{array}{ll}
\text { 1) } & \mathcal{C}\left(F\left(p_{k}, q_{k}\right)\right)=F\left(\mathcal{C}\left(p_{k}\right), \mathcal{C}\left(q_{k}\right)\right) \\
\text { 2) } & \mathcal{C}\left(\left\{F\left(p_{k}, q_{k}\right), G\left(p_{k}, q_{k}\right)\right\}\right)=\{\mathcal{C}(F), \mathcal{C}(G)\} \\
3) & \mathcal{C}\left(H\left(p_{k}, q_{k}\right)\right)=H\left(p_{k}, q_{k}\right)
\end{array}
$$

Here $F\left(p_{k}, q_{k}\right), G\left(p_{k}, q_{k}\right)$ and the Hamiltonian $H\left(p_{k}, q_{k}\right)$ are functionals on $\mathcal{M}$ depending analytically on the fields $q_{k}(x, t)$ and $p_{k}(x, t)$.

The complexification of the ATFT is rather straightforward. The resulting complex ATFT (CATFT) can be written down as standard Hamiltonian system with twice as many fields $\boldsymbol{q}^{a}(x, t), \boldsymbol{p}^{a}(x, t), a=0,1$ :

$$
\begin{aligned}
& \boldsymbol{p}^{\mathbb{C}}(x, t)=\boldsymbol{p}^{0}(x, t)+i \boldsymbol{p}^{1}(x, t), \quad \boldsymbol{q}^{\mathbb{C}}(x, t)=\boldsymbol{q}^{0}(x, t)+i \boldsymbol{q}^{1}(x, t), \\
& \left\{q_{k}^{0}(x, t), p_{j}^{0}(y, t)\right\}=-\left\{q_{k}^{1}(x, t), p_{j}^{1}(y, t)\right\}=\delta_{k j} \delta(x-y) .
\end{aligned}
$$

The densities of the corresponding Hamiltonian and symplectic form equal

$$
\begin{aligned}
\mathcal{H}_{\mathrm{ATFT}}^{\mathbb{C}} & \equiv \operatorname{Re} \mathcal{H}_{\mathrm{ATFT}}\left(\boldsymbol{p}^{0}+i \boldsymbol{p}^{1}, \boldsymbol{q}^{0}+i \boldsymbol{q}^{1}\right) \\
& =\frac{1}{2}\left(\boldsymbol{p}^{0}, \boldsymbol{p}^{0}\right)-\frac{1}{2}\left(\boldsymbol{p}^{1}, \boldsymbol{p}^{1}\right)+\sum_{k=0}^{r} e^{-\left(\boldsymbol{q}^{0}, \alpha_{k}\right)} \cos \left(\left(\boldsymbol{q}^{1}, \alpha_{k}\right)\right), \\
\omega^{\mathbb{C}} & =\left(d \boldsymbol{p}^{0} \wedge i d \boldsymbol{q}^{0}\right)-\left(d \boldsymbol{p}^{1} \wedge d \boldsymbol{q}^{1}\right) .
\end{aligned}
$$


The family of RHF then are obtained from the CATFT by imposing an invariance condition with respect to the involution $\tilde{\mathcal{C}} \equiv \mathcal{C} \circ *$ where by $*$ we denote the complex conjugation. The involution $\tilde{\mathcal{C}}$ splits the phase space $\mathcal{M}^{\mathbb{C}}$ into a direct sum $\mathcal{M}^{\mathbb{C}} \equiv \mathcal{M}_{+}^{\mathbb{C}} \oplus \mathcal{M}_{-}^{\mathbb{C}}$ where

$$
\mathcal{M}_{+}^{\mathbb{C}}=\mathcal{M}_{0} \oplus i \mathcal{M}_{1}, \quad \mathcal{M}_{-}^{\mathbb{C}}=i \mathcal{M}_{0} \oplus \mathcal{M}_{1},
$$

The phase space of the RHF is $\mathcal{M}_{\mathbb{R}} \equiv \mathcal{M}_{+}^{\mathbb{C}}$. By $\mathcal{M}_{0}$ and $\mathcal{M}_{1}$ we denote the eigensubspaces of $\mathcal{C}$, i.e. $\mathcal{C}\left(u_{a}\right)=(-1)^{a} u_{a}$ for any $u_{a} \in \mathcal{M}_{a}$.

Thus to each involution $\mathcal{C}$ satisfying 1 ) - 3) one can relate a RHF of the ATFT. Due to the condition 3) $\mathcal{C}$ must preserve the system of admissible roots of $\mathfrak{g}$; such involutions can be constructed from the $\mathbb{Z}_{2}$-symmetries of the extended Dynkin diagrams of $\mathfrak{g}$ studied in 6].

In fact one can relate ATFT models not only to the algebra $\operatorname{sl}(n)$ [2], but also to each Kac-Moody algebra 9, 10, 11]; for the theory of Kac-Moody algebras see [13. The construction is as follows. Let $\pi_{\mathfrak{g}}=\left\{\alpha_{0}, \alpha_{1}, \ldots, \alpha_{r}\right\}$ be the set of admissible roots of $\mathfrak{g}$. Then the corresponding ATFT model is given by:

$$
\begin{array}{rlrl}
H_{\mathfrak{g}} & =\int_{-\infty}^{\infty} d x \mathcal{H}_{\mathfrak{g}}(x, t), & \mathcal{H}_{\mathfrak{g}}(x, t)=\frac{1}{2}(\boldsymbol{p}(x, t), \boldsymbol{p}(x, t))+\sum_{k=0}^{r} n_{k} e^{-\left(\boldsymbol{q}(x, t), \alpha_{k}\right)}, \\
\Omega_{\mathfrak{g}}=\int_{-\infty}^{\infty} d x \omega_{\mathfrak{g}}(x, t), & \omega_{\mathfrak{g}}(x, t)=(\delta \boldsymbol{p}(x, t) \wedge \delta \boldsymbol{q}(x, t)),
\end{array}
$$

In what follows we will need also the integer coefficients $n_{k}$ that provide the decomposition of the $\alpha_{0}$ over the simple roots of $\mathfrak{g}$ :

$$
-\alpha_{0}=\sum_{k=1}^{r} n_{k} \alpha_{k} .
$$

Just like for the $\operatorname{sl}(n)$ case one can consider the complexification:

$$
\begin{aligned}
H_{\mathfrak{g}}^{\mathbb{C}} & =\int_{-\infty}^{\infty} d x \mathcal{H}_{\mathfrak{g}}^{\mathbb{C}}(x, t), \quad \mathcal{H}_{\mathfrak{g}}^{\mathbb{C}}(x, t)=\frac{1}{2}\left(\boldsymbol{p}^{0}(x, t), \boldsymbol{p}^{0}(x, t)\right)-\frac{1}{2}\left(\boldsymbol{p}^{1}(x, t), \boldsymbol{p}^{1}(x, t)\right) \\
& +\sum_{k=0}^{r} n_{k} e^{-\left(\boldsymbol{q}^{0}(x, t), \alpha_{k}\right)} \cos \left(\boldsymbol{q}^{1}(x, t), \alpha_{k}\right), \\
\Omega_{\mathfrak{g}}^{\mathbb{C}} & =\int_{-\infty}^{\infty} d x \omega_{\mathfrak{g}}^{\mathbb{C}}(x, t), \quad \omega_{\mathfrak{g}}^{\mathbb{C}}=\left(\delta \boldsymbol{p}^{0}(x, t) \wedge \delta \boldsymbol{q}^{0}(x, t)-\left(\delta \boldsymbol{p}^{1}(x, t) \wedge \delta \boldsymbol{q}^{1}(x,(\{3) .13)\right.\right.
\end{aligned}
$$

In order to construct the RHF of the ATFT we will make use of specifically constructed involutions $\mathcal{C}$ of the phase space $\mathcal{M}_{\mathfrak{g}} \equiv\{\boldsymbol{p}(x, t), \boldsymbol{q}(x, t)\}$. An important fact in our construction will be that to each involution $\mathcal{C}$ there exist a dual involution (involutive automorphism) $\mathcal{C}^{\#}$ of $\mathfrak{g}$. Property 3) above along with the definition (3.9) of $\mathcal{H}_{\mathfrak{g}}$ implies that $\mathcal{C}^{\#}$ preserves the set of admissible roots $\pi_{\mathfrak{g}}$. For brevity below we will skip the dependence of $\boldsymbol{p}$ and $\boldsymbol{q}$ on $x$ and $t$.

Indeed, the condition 3) above requires that:

$$
(\mathcal{C}(\boldsymbol{q}), \alpha)=\left(\boldsymbol{q}, \mathcal{C}^{\#}(\alpha)\right), \quad \alpha \in \pi_{\mathfrak{g}}
$$

and therefore we must have $\mathcal{C}\left(\pi_{\mathfrak{g}}\right)=\pi_{\mathfrak{g}}$. 
The relation (3.14) defines uniquely the relation between $\mathcal{C}$ and $\mathcal{C}^{\#}$. Using $\mathcal{C}^{\#}$ we can split the root space $\mathbb{E}^{n}$ into direct sum $\mathbb{E}^{n}=\mathbb{E}_{+} \oplus \mathbb{E}_{-}$of two eigensubspaces of $\mathcal{C}^{\#}$. Taking the average of the roots $\alpha_{j}$ with respect to $\mathcal{C}^{\#}$ we get:

$$
\beta_{j}=\frac{1}{2}\left(\alpha_{j}+\mathcal{C}^{\#}\left(\alpha_{j}\right)\right), \quad j=0, \ldots, n_{+}=\operatorname{dim} \mathbb{E}_{+} .
$$

By construction the set $\left\{\beta_{0}, \beta_{1}, \ldots, \beta_{n_{+}}\right\}$will be a set of admissible roots for some KacMoody algebra with rank $n_{+}$. Graphically each set of admissible roots can be represented by an extended Dynkin diagrams. Therefore one can relate an automorphism $\mathcal{C}^{\#}$ to each $\mathbb{Z}_{2}$ symmetry of the extended Dynkin diagram.

The splitting of $\mathbb{E}^{n}$ naturally leads to the splittings of the fields:

$$
\boldsymbol{p}=\boldsymbol{p}^{+}+\boldsymbol{p}^{-}, \quad \boldsymbol{q}=\boldsymbol{q}^{+}+\boldsymbol{q}^{-},
$$

where $\boldsymbol{p}^{+}, \boldsymbol{q}^{+} \in \mathbb{E}_{+}$and $\boldsymbol{p}^{-}, \boldsymbol{q}^{-} \in \mathbb{E}_{-}$. If we also introduce:

$$
\gamma_{j}=\frac{1}{2}\left(\alpha_{j}-\mathcal{C}^{\#}\left(\alpha_{j}\right)\right), \quad j=0, \ldots, n_{-}=\operatorname{dim} \mathbb{E}_{-} .
$$

Remark 2. In enumerating the vectors $\beta_{j}$ and $\gamma_{j}$ we take into account only those values of $j$ which lead to non-vanishing results in the r.h.sides of (3.15) and (3.17).

Then applying the ideas of [1] we obtain the following result for the RHF of the ATFT related to $\mathfrak{g}$ :

$$
\begin{aligned}
\mathcal{H}_{\mathfrak{g}}^{\mathbb{R}} & =\frac{1}{2}\left(\boldsymbol{p}^{+}, \boldsymbol{p}^{+}\right)-\frac{1}{2}\left(\boldsymbol{p}^{-}, \boldsymbol{p}^{-}\right)+\sum_{k=0}^{r} n_{k}^{\prime} e^{-\left(\boldsymbol{q}^{+}, \beta_{k}\right)} \cos \left(\boldsymbol{q}^{-}, \gamma_{j}\right), \\
\omega_{\mathfrak{g}}^{\mathbb{R}} & =\left(\delta \boldsymbol{p}^{+} \wedge \delta \boldsymbol{q}^{+}\right)-\left(\delta \boldsymbol{p}^{-} \wedge \delta \boldsymbol{q}^{-}\right),
\end{aligned}
$$

where $n_{k}^{\prime}$ are the minimal positive integers for which

$$
-\beta_{0}=\sum_{k=1}^{r} n_{k}^{\prime} \beta_{k} .
$$

We can consider particular cases of the models (3.18) by imposing on them the constraints $\boldsymbol{p}^{-}=0$ and $\boldsymbol{q}^{-}=0$. This leads to an ATFT related to a Kac-Moody algebra $\mathfrak{g}^{\prime}$ of rank $r^{\prime}$ whose system of admissible roots $\pi^{\prime}=\left\{\beta_{0}, \beta_{1}, \ldots, \beta_{r^{\prime}}\right\}$ is obtained from $\pi$ by averaging with respect to the involution $\mathcal{C}$. For each of the examples listed in the next Section we provide both sets of admissible roots.

The RHF of ATFT are more general integrable systems than the models described in [11, 6] which involve only the fields $\boldsymbol{q}^{+}, \boldsymbol{p}^{+}$invariant with respect to $\mathcal{C}$.

\section{Examples}

In this Section we provide several examples of RHF of ATFT related to Kac-Moody algebras from the series $\boldsymbol{A}_{n}^{(a)}$ and $\boldsymbol{D}_{n}^{(a)}$ where the height $a$ can be either 1 or 2 . We show that the involutions $\mathcal{C}$ in all these cases are dual to $\mathbb{Z}_{2}$-symmetries of the extended Dynkin diagrams derived in $[6$. 
The dynamical variables for the corresponding ATFT systems related to the type $\boldsymbol{A}_{r}$ Kac-Moody algebras are provided as $r+1$-component vector-valued functions $\boldsymbol{p}(x, t)$ and $\boldsymbol{q}(x, t)$ restricted by the condition

$$
\sum_{k=1}^{r+1} p_{k}(x, t)=\sum_{k=1}^{r+1} q_{k}(x, t)=0 .
$$

This is related to the fact that the root systems of type $\boldsymbol{A}_{r}$ Kac-Moody algebras are conveniently embedded as $r$-dimensional subspace of $\mathbb{E}^{r+1}$ orthogonal to the vector $e_{1}+$ $e_{2}+\cdots+e_{r+1}$.

Below we list several examples which illustrate the procedure outlined above and display new types of ATFT.

Example 1. We choose $\mathfrak{g} \simeq \boldsymbol{A}_{2 r-1}^{(1)}$ and fix up the involution $\mathcal{C}$ by:

$$
\mathcal{C}\left(q_{k}\right)=-q_{2 r+1-k}, \quad \mathcal{C}\left(p_{k}\right)=-p_{2 r+1-k}, \quad k=1, \ldots, r,
$$

The coordinates in $\mathcal{M}_{ \pm}$are given by:

$$
q_{k}^{ \pm}=\frac{1}{\sqrt{2}}\left(q_{k} \mp q_{2 r+1-k}\right), \quad p_{k}^{ \pm}=\frac{1}{\sqrt{2}}\left(p_{k} \mp p_{2 r+1-k}\right),
$$

where $k=1, \ldots, r$, i.e., $\operatorname{dim} \mathcal{M}_{+}=2 r, \operatorname{dim} \mathcal{M}_{-}=2 r-2$.

This involution induces an involution $\mathcal{C}^{\#}$ of the Kac-Moody algebra $\boldsymbol{A}_{2 r-1}^{(1)}$ which acts on the root space as follows, (see fig. 1):

$$
\begin{array}{rr}
\mathcal{C}^{\#} e_{k}=-e_{2 r+1-k}, \quad k=1, \ldots, 2 r ; \\
\mathcal{C}^{\#} \alpha_{k}=\alpha_{2 r-k}, \quad k=1, \ldots r-1 ; \quad \mathcal{C}^{\#} \alpha_{0}=\alpha_{0}, & \mathcal{C}^{\#} \alpha_{r}=\alpha_{r} .
\end{array}
$$

The involution $\mathcal{C}^{\#}$ splits the root space $\mathbb{E}^{2 r-1}$ into a direct sum of its eigensubspaces: $\mathbb{E}^{2 r-1}=\mathbb{E}_{+} \oplus \mathbb{E}_{-}$with $\operatorname{dim} \mathbb{E}_{+}=r$ and $\operatorname{dim} \mathbb{E}_{-}=r-1$. The restriction of $\pi$ onto $\mathbb{E}_{+}$ leads to the admissible root system $\pi^{\prime}=\left\{\beta_{0}, \ldots, \beta_{r}\right\}$ of $\boldsymbol{C}_{r}^{(1)}$ :

$$
\beta_{k}=\frac{1}{2}\left(\alpha_{k}+\mathcal{C}^{\#} \alpha_{k}\right), \quad k=1, \ldots, r-1 ; \quad \beta_{0}=\alpha_{0}, \quad \beta_{r}=\alpha_{r} .
$$

The subspace $\mathbb{E}_{-}$is spanned by

$$
\gamma_{k}=\frac{1}{2}\left(\alpha_{k}-\mathcal{C}^{\#} \alpha_{k}\right), \quad k=1, \ldots, r-1 .
$$

Then the densities $\mathcal{H}_{1}^{\mathbb{R}}, \omega_{1}^{\mathbb{R}}$ for the RHF of AFTF equal:

$$
\begin{aligned}
\mathcal{H}_{1}^{\mathbb{R}} & =\frac{1}{2} \sum_{k=1}^{r-1}\left(p_{k}^{+2}-p_{k}^{-2}\right)+\sum_{k=1}^{r-1} 2 e^{\left(q_{k+1}^{+}-q_{k}^{+}\right) / \sqrt{2}} \cos \left(\frac{q_{k+1}^{-}-q_{k}^{-}}{\sqrt{2}}\right) \\
& +e^{\sqrt{2} q_{1}^{+}}+e^{-\sqrt{2} q_{r}^{+}}, \\
\omega_{1}^{\mathbb{R}} & =\sum_{k=1}^{r} \delta p_{k}^{+}(x) \wedge \delta q_{k}^{+}(x)-\sum_{k=1}^{r+1} d p_{k}^{-} \wedge d q_{k}^{-} .
\end{aligned}
$$

where $\boldsymbol{p}_{k}^{ \pm}=d \boldsymbol{q}_{k}^{ \pm} / d t$. If we put $\boldsymbol{q}^{-}=0$ and $\boldsymbol{p}^{-}=0$ we get the reduced ATFT related to the Kac-Moody algebra $\boldsymbol{C}_{r+1}^{(1)}[6]$. 


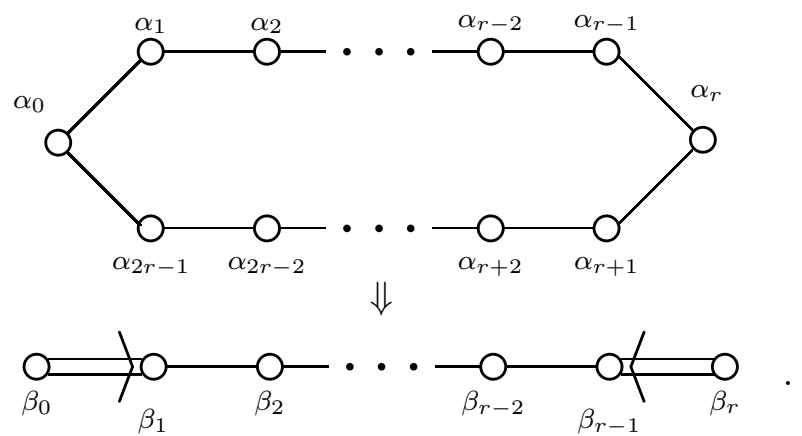

Figure 1. $\boldsymbol{A}_{2 r-1}^{(1)} \rightarrow \boldsymbol{C}_{r}^{(1)}$

Example 2. Choose $\mathfrak{g} \simeq \boldsymbol{A}_{2 r+1}^{(1)}, r>2$ and fix up the involution $\mathcal{C}$ by:

$$
\begin{aligned}
& \mathcal{C}\left(q_{k}\right)=-q_{2 r+2-k}, \quad \mathcal{C}\left(p_{k}\right)=-p_{2 r+2-k}, \quad k=1, \ldots, r, \\
& \mathcal{C}\left(q_{r+1}\right)=-q_{r+1}, \quad \mathcal{C}\left(p_{r+1}\right)=-p_{r+1} .
\end{aligned}
$$

Here the coordinates in $\mathcal{M}_{ \pm}$are given by:

$$
\begin{array}{r}
q_{k}^{ \pm}=\frac{1}{\sqrt{2}}\left(q_{k} \mp q_{2 r+2-k}\right), \quad p_{k}^{ \pm}=\frac{1}{\sqrt{2}\left(p_{k} \mp p_{2 r+2-k}\right),} \quad k=1, \ldots, r, \\
q_{r+1}^{-}=q_{r+1}, \quad p_{r+1}^{-}=p_{r+1},
\end{array}
$$

i.e., $\operatorname{dim} \mathcal{M}_{+}=2 r$ and $\operatorname{dim} \mathcal{M}_{-}=2 r+2$.

This involution induces an involution $\mathcal{C}^{\#}$ of the Kac-Moody algebra $\boldsymbol{A}_{2 r+1}^{(1)}$ which acts on the root space as follows (see fig. 2):

$$
\begin{array}{r}
\mathcal{C}^{\#} e_{k}=-e_{2 r+2-k}, \quad k=1, \ldots, r ; \quad k=r+2, \ldots, 2 r+1 ; \\
\mathcal{C}^{\#} e_{2 r+2}=-e_{2 r+2}, \quad \mathcal{C}^{\#} e_{r+1}=-e_{r+1}, \\
\mathcal{C}^{\#} \alpha_{k}=\alpha_{2 r+1-k}, \quad k=0, \ldots 2 r+1 ;
\end{array}
$$

The involution $\mathcal{C}^{\#}$ splits the root space $\mathbb{E}^{2 r}$ into a direct sum of its eigensubspaces: $\mathbb{E}^{2 r+1}=$ $\mathbb{E}_{+} \oplus \mathbb{E}_{-}$with $\operatorname{dim} \mathbb{E}_{+}=r$ and $\operatorname{dim} \mathbb{E}_{-}=r+1$. The restriction of $\pi$ onto $\mathbb{E}_{+}$leads to the admissible root system $\pi^{\prime}=\left\{\beta_{0}, \ldots, \beta_{r}\right\}$ of $\boldsymbol{D}_{r+1}^{(2)}$ :

$$
\beta_{k}=\frac{1}{2}\left(\alpha_{k}+\mathcal{C}^{\#} \alpha_{k}\right), \quad k=0, \ldots, r
$$

The subspace $\mathbb{E}_{-}$is spanned by

$$
\gamma_{k}=\frac{1}{2}\left(\alpha_{k}-\mathcal{C}^{\#} \alpha_{k}\right), \quad k=0, \ldots, r
$$




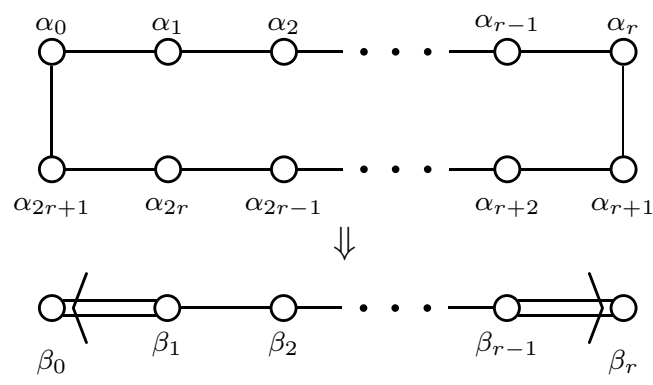

Figure 2. $\quad \boldsymbol{A}_{2 r+1}^{(1)} \rightarrow \boldsymbol{D}_{r+1}^{(2)}$

Then the densities $\mathcal{H}_{2}^{\mathbb{R}}, \omega_{2}^{\mathbb{R}}$ for the RHF of AFTF equal:

$$
\begin{aligned}
& \mathcal{H}_{2}^{\mathbb{R}}=\frac{1}{2} \sum_{k=1}^{r} p_{k}^{+2}-\frac{1}{2} \sum_{k=1}^{r+1} p_{k}^{-2}+2 e^{-q_{r-1}^{+} / \sqrt{2}} \cos \left(\frac{q_{r-1}^{-}}{\sqrt{2}}-q_{r+1}^{-}\right) \\
& +\sum_{k=1}^{r-1} 2 e^{\left(q_{k+1}^{+}-q_{k}^{+}\right) / \sqrt{2}} \cos \left(\frac{q_{k+1}^{-}-q_{k}^{-}}{\sqrt{2}}\right)+2 e^{q_{1}^{+} / \sqrt{2}} \cos \left(\frac{q_{1}^{-}}{\sqrt{2}}-q_{r+1}^{-}\right) . \\
& \omega_{2}^{\mathbb{R}}=\sum_{k=1}^{r} \delta p_{k}^{+} \wedge \delta q_{k}^{+}-\sum_{k=1}^{r+1} \delta p_{k}^{-} \wedge \delta q_{k}^{-}
\end{aligned}
$$

where $\boldsymbol{p}_{k}^{ \pm}=d \boldsymbol{q}_{k}^{ \pm} / d t$. If we put $q_{j}^{-}=0$ and $p_{j}^{-}=0$ we get the reduced ATFT related to the Kac-Moody algebra $\boldsymbol{D}_{r+1}^{(2)}[6]$.

Example 3. Now choose $\mathfrak{g} \simeq \boldsymbol{A}_{2 r}^{(1)}$ and fix up the involution $\mathcal{C}$ by:

$$
\begin{aligned}
& \mathcal{C}\left(q_{k}\right)=-q_{2 r+2-k}, \quad \mathcal{C}\left(p_{k}\right)=-p_{2 r+2-k}, \quad k=1, \ldots, r-1, \\
& \mathcal{C}\left(q_{r}\right)=-q_{r}, \quad \mathcal{C}\left(p_{r}\right)=-p_{r} .
\end{aligned}
$$

The coordinates in $\mathcal{M}_{ \pm}$:

$$
\begin{array}{r}
q_{k}^{ \pm}=\frac{1}{\sqrt{2}}\left(q_{k} \mp q_{2 r+2-k}\right), \quad p_{k}^{ \pm}=\frac{1}{\sqrt{2}}\left(p_{k} \mp p_{2 r+2-k}\right), k=1, \ldots, r-1 ; \\
q_{r}^{-}=q_{r}, \quad p_{r}^{-}=p_{r},
\end{array}
$$

i.e., $\operatorname{dim} \mathcal{M}_{+}=2 r-2$ and $\operatorname{dim} \mathcal{M}_{-}=2 r$.

This involution induces an involution $\mathcal{C}^{\#}$ of the Kac-Moody algebra $\boldsymbol{A}_{2 r}^{(1)}$ which acts on the root space as follows (see fig. [3):

$$
\begin{array}{r}
\mathcal{C}^{\#} e_{k}=-e_{2 r+2-k}, \quad k=1, \ldots, r ; \quad k=r+2, \ldots, 2 r+1 ; \\
\mathcal{C}^{\#} \alpha_{k}=\alpha_{2 r+1-k}, \quad k=1, \ldots r ; \quad \mathcal{C}^{\#} \alpha_{0}=\alpha_{0},
\end{array}
$$

The involution $\mathcal{C}^{\#}$ splits the root space $\mathbb{E}^{2 r}$ into a direct sum of its eigensubspaces: $\mathbb{E}^{2 r}=$ $\mathbb{E}_{+} \oplus \mathbb{E}_{-}$with $\operatorname{dim} \mathbb{E}_{+}=r-1$ and $\operatorname{dim} \mathbb{E}_{-}=r$. The restriction of $\pi$ onto $\mathbb{E}_{+}$leads to the admissible root system $\pi^{\prime}=\left\{\beta_{0}, \ldots, \beta_{r}\right\}$ of $\boldsymbol{A}_{2 r}^{(2)}$ :

$$
\beta_{k}=\frac{1}{2}\left(\alpha_{k}+\mathcal{C}^{\#} \alpha_{k}\right), \quad k=1, \ldots, r-1 ; \quad \beta_{0}=\alpha_{0}, \quad \beta_{r}=\alpha_{r} .
$$


The subspace $\mathbb{E}_{-}$is spanned by

$$
\gamma_{k}=\frac{1}{2}\left(\alpha_{k}-\mathcal{C}^{\#} \alpha_{k}\right), \quad k=1, \ldots, r
$$

Then the densities $\mathcal{H}_{3}^{\mathbb{R}}, \omega_{3}^{\mathbb{R}}$ for the RHF of AFTF equal:

$$
\begin{aligned}
& \mathcal{H}_{3}^{\mathbb{R}}=\frac{1}{2} \sum_{k=1}^{r-1} p_{k}^{+2}-\frac{1}{2} \sum_{k=1}^{r} p_{k}^{-2}+2 e^{-q_{r-1}^{+} / \sqrt{2}} \cos \left(\frac{q_{r-1}^{-}}{\sqrt{2}}-q_{r+1}^{-}\right) \\
& +\sum_{k=1}^{r-1} 2 e^{\left(q_{k+1}^{+}-q_{k}^{+}\right) / \sqrt{2}} \cos \left(\frac{q_{k+1}^{-}-q_{k}^{-}}{\sqrt{2}}\right)+2 e^{q_{1}^{+} / \sqrt{2}} \cos \left(\frac{q_{1}^{-}}{\sqrt{2}}-q_{r+1}^{-}\right) . \\
& \omega_{3}^{\mathbb{R}}=\sum_{k=1}^{r-1} \delta p_{k}^{+} \wedge \delta q_{k}^{+}-\sum_{k=1}^{r} \delta p_{k}^{-} \wedge \delta q_{k}^{-}
\end{aligned}
$$

where $\boldsymbol{p}_{k}^{ \pm}=d \boldsymbol{q}_{k}^{ \pm} / d x$. If we put $q_{j}^{-}=0$ and $p_{j}^{-}=0$ we get the reduced ATFT related to the Kac-Moody algebra $\boldsymbol{A}_{2 r}^{(2)}[6]$.

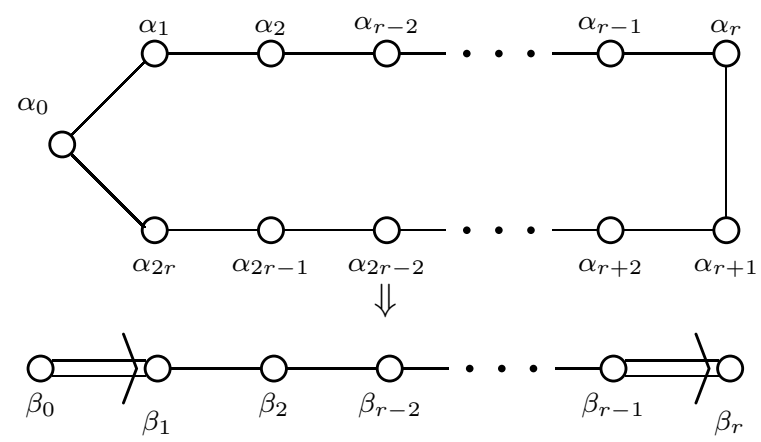

Figure 3. $\boldsymbol{A}_{2 r}^{(1)} \rightarrow \boldsymbol{A}_{2 r}^{(2)}$

Example 4. Let us choose now $\mathfrak{g} \simeq \mathbf{D}_{r+1}^{(1)}$ and fix up the involution $\mathcal{C}$ acting on the coordinates in the phase space by:

$$
\mathcal{C}\left(q_{k}\right)=q_{k}, \quad \mathcal{C}\left(p_{k}\right)=p_{k}, \quad k=1, \ldots, r-1 ; \quad \mathcal{C}\left(q_{r}\right)=-q_{r}, \quad \mathcal{C}\left(p_{r}\right)=-p_{r}
$$

Then introducing on $\mathcal{M}_{ \pm}$new coordinates by

$$
\begin{aligned}
& q_{k}^{+}=q_{k}, \quad p_{k}^{+}=p_{k}, \quad q_{r}^{-}=q_{r}, \quad p_{r}^{-}=p_{r}, \quad k=1, \ldots, r \\
& q_{r+1}^{-}=q_{r+1}, \quad p_{r+1}^{-}=p_{r+1},
\end{aligned}
$$

i.e. $\operatorname{dim} \mathcal{M}_{+}=2 r$ and $\operatorname{dim} \mathcal{M}_{-}=2$.

This involution induces an involution $\mathcal{C}^{\#}$ of the Kac-Moody algebra $\boldsymbol{D}_{r+1}^{(1)}$ which acts on the root space as follows (see fig. 4):

$$
\begin{array}{cc}
\mathcal{C}^{\#} e_{k}=e_{k}, & k=1, \ldots, r ; \quad \mathcal{C}^{\#} e_{r+1}=-e_{r+1}, \\
\mathcal{C}^{\#} \alpha_{k}=\alpha_{k}, & \mathcal{C}^{\#} \alpha_{r+1}=\alpha_{r}, \quad \mathcal{C}^{\#} \alpha_{r}=\alpha_{r+1},
\end{array}
$$


The involution $\mathcal{C}^{\#}$ splits the root space $\mathbb{E}^{r+1}$ into a direct sum of its eigensubspaces: $\mathbb{E}^{r+1}=\mathbb{E}_{+} \oplus \mathbb{E}_{-}$with $\operatorname{dim} \mathbb{E}_{+}=r$ and $\operatorname{dim} \mathbb{E}_{-}=1$. The restriction of $\pi$ onto $\mathbb{E}_{+}$leads to the admissible root system $\pi^{\prime}=\left\{\beta_{0}, \ldots, \beta_{r}\right\}$ of $\boldsymbol{B}_{r}^{(1)}$ :

$$
\beta_{k}=\alpha_{k}, \quad k=0, \ldots, r-1 ; \quad \beta_{r}=\frac{1}{2}\left(\alpha_{r}+\mathcal{C}^{\#} \alpha_{r}\right),
$$

The subspace $\mathbb{E}_{-}$is spanned by the only nontrivial vector

$$
\gamma_{r}=\frac{1}{2}\left(\alpha_{r}-\mathcal{C}^{\#} \alpha_{r}\right)=-e_{r+1},
$$

The RHF is described by the following densities $\mathcal{H}_{4}^{\mathbb{R}}, \omega_{4}^{\mathbb{R}}$ :

$$
\begin{aligned}
& \mathcal{H}_{4}^{\mathbb{R}}=\frac{1}{2} \sum_{k=1}^{r} p_{k}^{+2}-\frac{1}{2} p_{r+1}^{-}{ }^{2}+\sum_{k=0}^{r-1} e^{q_{k+1}^{+}-q_{k}^{+}}+2 e^{q_{r-1}^{+}} \cos q_{r+1}^{-}, \\
& \omega_{4}^{\mathbb{R}}=\sum_{k=1}^{r} \delta p_{k}^{+} \wedge \delta q_{k}^{+}-\delta p_{r+1}^{-} \wedge \delta q_{r+1}^{-} .
\end{aligned}
$$
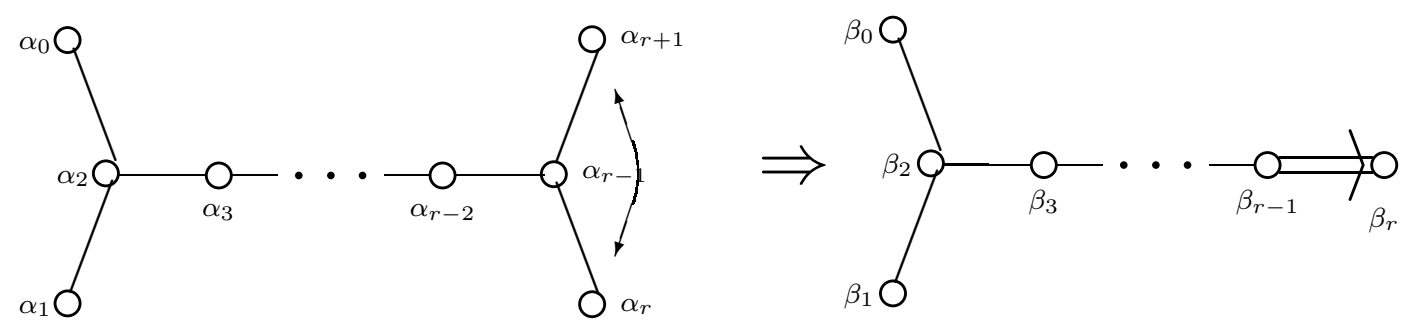

Figure 4. Reductions of $\boldsymbol{D}_{r+1}^{(1)}$ to $\boldsymbol{B}_{r}^{(1)}$.

Example 5. Next we choose $\mathfrak{g} \simeq \mathbf{D}_{r+2}^{(1)}$ and fix up the involution $\mathcal{C}$ acting on the coordinates in the phase space by:

$$
\begin{array}{r}
\mathcal{C}\left(q_{k}\right)=q_{k}, \quad \mathcal{C}\left(p_{k}\right)=p_{k}, \quad k=2, \ldots, r+1 ; \\
\mathcal{C}\left(q_{1}\right)=-q_{1}, \quad \mathcal{C}\left(p_{1}\right)=-p_{1}, \quad \mathcal{C}\left(q_{r+2}\right)=-q_{r+2}, \quad \mathcal{C}\left(p_{r+2}\right)=-p_{r+2} .
\end{array}
$$

Then introducing on $\mathcal{M}_{ \pm}$new coordinates by

$$
\begin{array}{ll}
q_{k}^{+}=q_{k}, & p_{k}^{+}=p_{k}, \quad q_{k}^{-}=0, \quad p_{k}^{-}=0, \quad k=2, \ldots, r+1 ; \\
q_{1}^{-}=q_{1}, & p_{1}^{-}=p_{1}, \quad q_{r+2}^{-}=q_{r+2}, \quad p_{r+2}^{-}=p_{r+2},
\end{array}
$$

i.e. $\operatorname{dim} \mathcal{M}_{+}=2 r$ and $\operatorname{dim} \mathcal{M}_{-}=4$.

This involution induces an involution $\mathcal{C}^{\#}$ of the Kac-Moody algebra $\boldsymbol{D}_{r+2}^{(1)}$ which acts on the root space as follows (see fig. [5):

$$
\begin{array}{r}
\mathcal{C}^{\#} e_{k}=e_{k}, \quad k=2, \ldots, r ; \quad \mathcal{C}^{\#} e_{1}=-e_{1}, \quad \mathcal{C}^{\#} e_{r+1}=-e_{r+1}, \\
\mathcal{C}^{\#} \alpha_{k}=\alpha_{k}, \quad \mathcal{C}^{\#} \alpha_{r+1}=\alpha_{r}, \quad \mathcal{C}^{\#} \alpha_{r}=\alpha_{r+1}, \\
\mathcal{C}^{\#} \alpha_{1}=\alpha_{0}, \quad \mathcal{C}^{\#} \alpha_{0}=\alpha_{1},
\end{array}
$$




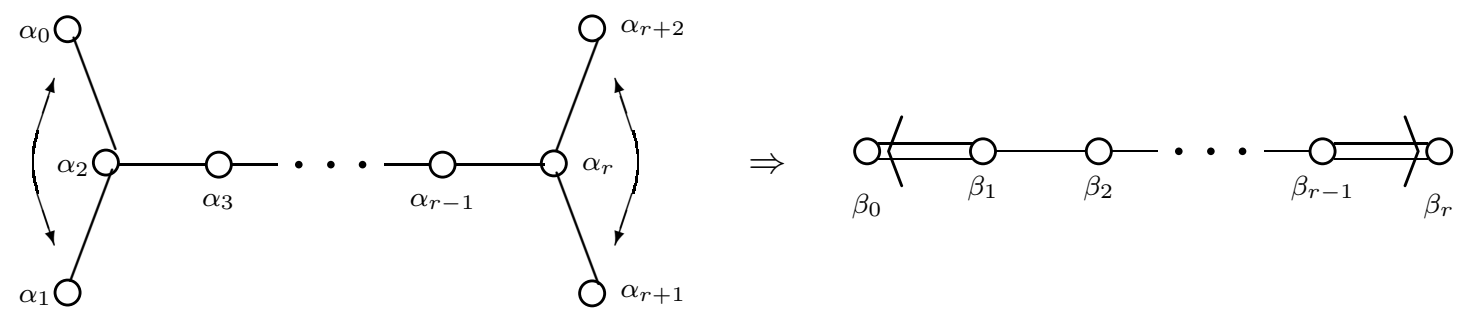

Figure 5. Reductions of $\boldsymbol{D}_{r+2}^{(1)}$ to $\boldsymbol{D}_{r+1}^{(2)}$.

The involution $\mathcal{C}^{\#}$ splits the root space $\mathbb{E}^{r+2}$ into a direct sum of its eigensubspaces: $\mathbb{E}^{r+2}=\mathbb{E}_{+} \oplus \mathbb{E}_{-}$with $\operatorname{dim} \mathbb{E}_{+}=r$ and $\operatorname{dim} \mathbb{E}_{-}=2$. The restriction of $\pi$ onto $\mathbb{E}_{+}$leads to the admissible root system $\pi^{\prime}=\left\{\beta_{0}, \ldots, \beta_{r}\right\}$ of $\boldsymbol{D}_{r+1}^{(2)}$ :

$$
\beta_{k}=\alpha_{k}, \quad k=1, \ldots, r-1 ; \quad \beta_{j}=\frac{1}{2}\left(\alpha_{j}+\mathcal{C}^{\#} \alpha_{j}\right), \quad j=0, r+1 .
$$

The subspace $\mathbb{E}_{-}$is spanned by the vectors

$$
\gamma_{r+1}=\frac{1}{2}\left(\alpha_{r}-\mathcal{C}^{\#} \alpha_{r}\right)=-e_{r+2}, \quad \gamma_{1}=\frac{1}{2}\left(\alpha_{1}-\mathcal{C}^{\#} \alpha_{0}\right)=e_{1},
$$

The RHF is described by the following densities $\mathcal{H}_{5}^{\mathbb{R}}, \omega_{5}^{\mathbb{R}}$ :

$$
\begin{aligned}
& \mathcal{H}_{5}^{\mathbb{R}}=\frac{1}{2} \sum_{k=2}^{r+1} p_{k}^{+2}-\frac{1}{2}\left(p_{1}^{-2}+p_{r+2}^{-}{ }^{2}\right)+\sum_{k=1}^{r} 2 e^{-\left(\boldsymbol{q}_{+}, \beta_{k}\right)}+e^{-\left(\boldsymbol{q}_{+}, \beta_{0}\right)} \cos q_{1}^{-} \\
& +e^{-\left(\boldsymbol{q}_{+}, \beta_{r}\right)} \cos q_{r+2}^{-}, \\
& \omega_{5}^{\mathbb{R}}=\sum_{k=2}^{r+1} \delta p_{k}^{+} \wedge \delta q_{k}^{+}-\delta p_{1}^{-} \wedge \delta q_{1}^{-}-\delta p_{r+2}^{-} \wedge \delta q_{r+2}^{-} .
\end{aligned}
$$

Example 6. Next we choose $\mathfrak{g} \simeq \mathbf{D}_{2 r}^{(1)}, r>3$ and fix up the involution $\mathcal{C}$ acting on the coordinates in the phase space by:

$$
\mathcal{C}\left(q_{k}\right)=-q_{2 r+1-k}, \quad \mathcal{C}\left(p_{k}\right)=-p_{2 r+1-k}, \quad k=1, \ldots, r .
$$

Then introducing on $\mathcal{M}_{ \pm}$new coordinates by

$$
\begin{array}{lll}
q_{k}^{+}=\frac{1}{\sqrt{2}}\left(q_{k}-q_{2 r+1-k}\right), & p_{k}^{+}=\frac{1}{\sqrt{2}}\left(p_{k}-p_{2 r+1-k}\right), & k=1, \ldots, r ; \\
q_{k}^{-}=\frac{1}{\sqrt{2}}\left(q_{k}+q_{2 r+1-k}\right), & p_{k}^{-}=\frac{1}{\sqrt{2}}\left(p_{k}+p_{2 r+1-k}\right), & k=1, \ldots, r ;
\end{array}
$$

i.e. $\operatorname{dim} \mathcal{M}_{+}=2 r$ and $\operatorname{dim} \mathcal{M}_{-}=2 r$. 

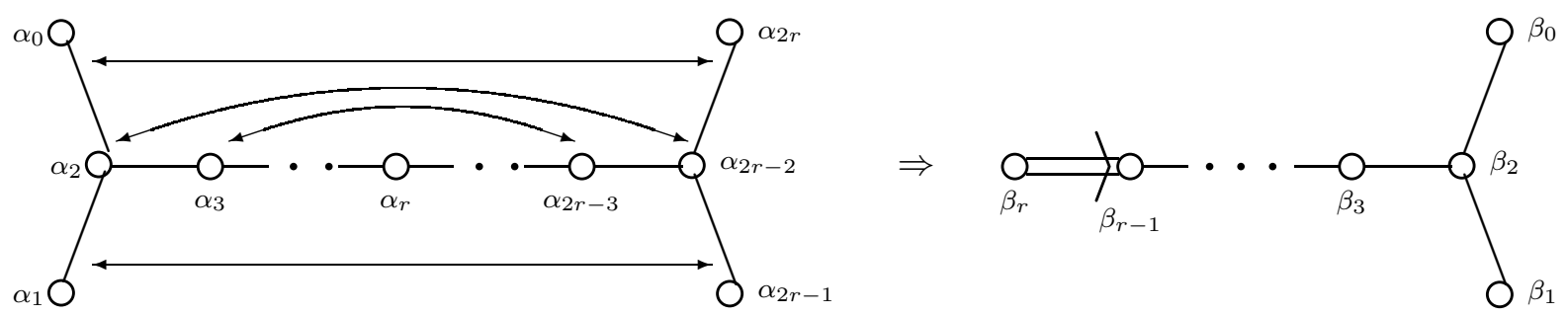

Figure 6. Reductions of $\boldsymbol{D}_{2 r}^{(1)}$ to $\boldsymbol{A}_{2 r-1}^{(2)}$.

This involution induces an involution $\mathcal{C}^{\#}$ of the Kac-Moody algebra $\boldsymbol{D}_{2 r}^{(1)}$ which acts on the root space as follows (see fig. 6):

$$
\begin{array}{r}
\mathcal{C}^{\#} e_{k}=-e_{2 r+1-k}, \quad k=1, \ldots, 2 r ; \\
\mathcal{C}^{\#} \alpha_{k}=\alpha_{2 r-k}, \quad k=1, \ldots, 2 r .
\end{array}
$$

The involution $\mathcal{C}^{\#}$ splits the root space $\mathbb{E}^{2 r}$ into a direct sum of its eigensubspaces: $\mathbb{E}^{2 r}=$ $\mathbb{E}_{+} \oplus \mathbb{E}_{-}$with $\operatorname{dim} \mathbb{E}_{+}=r$ and $\operatorname{dim} \mathbb{E}_{-}=r$. The restriction of $\pi$ onto $\mathbb{E}_{+}$leads to the admissible root system $\pi^{\prime}=\left\{\beta_{0}, \ldots, \beta_{r}\right\}$ of $\boldsymbol{A}_{2 r-1}^{(2)}$ :

$$
\beta_{k}=\frac{1}{2}\left(\alpha_{k}+\mathcal{C}^{\#} \alpha_{k}\right), \quad k=1, \ldots, r-1 ; \quad \beta_{r}=\alpha_{r} .
$$

The subspace $\mathbb{E}_{-}$is spanned by the vectors

$$
\gamma_{k}=\frac{1}{2}\left(\alpha_{k}-\mathcal{C}^{\#} \alpha_{k}\right), \quad k=1, \ldots, r-1 .
$$

The RHF is described by the following densities $\mathcal{H}_{6}^{\mathbb{R}}, \omega_{6}^{\mathbb{R}}$ :

$$
\begin{aligned}
& \mathcal{H}_{6}^{\mathbb{R}}=\frac{1}{2} \sum_{k=2}^{r+1}\left(p_{k}^{+2}-p_{k}^{+2}\right)+\sum_{k=1}^{r-1} 2 e^{-\left(\boldsymbol{q}_{+}, \beta_{k}\right)} \cos \left(\boldsymbol{q}_{-}, \gamma_{k}\right) \\
& +e^{-\left(\boldsymbol{q}_{+}, \beta_{0}\right)} \cos \left(\boldsymbol{q}_{-}, \gamma_{0}\right)+e^{-\left(\boldsymbol{q}_{+}, \beta_{1}\right)} \cos \left(\boldsymbol{q}_{-}, \gamma_{1}\right)+e^{-\left(\boldsymbol{q}_{+}, \beta_{r}\right)} \cos \left(\boldsymbol{q}_{-}, \gamma_{r}\right), \\
& \omega_{6}^{\mathbb{R}}=\sum_{k=1}^{r}\left(\delta p_{k}^{+} \wedge \delta q_{k}^{+}-\delta p_{k}^{-} \wedge \delta q_{k}^{-}\right) .
\end{aligned}
$$

Example 7. Finally we choose $\mathfrak{g} \simeq \mathbf{D}_{2 r+1}^{(1)}, r>3$ and fix up the involution $\mathcal{C}$ acting on the coordinates in the phase space by:

$$
\begin{array}{r}
\mathcal{C}\left(q_{k}\right)=-q_{2 r+2-k}, \quad \mathcal{C}\left(p_{k}\right)=-p_{2 r+2-k}, \quad k=1, \ldots, r ; \\
\mathcal{C}\left(q_{r+1}\right)=-q_{r+1}, \quad \mathcal{C}\left(p_{r+1}\right)=-p_{r+1} .
\end{array}
$$

Then introducing on $\mathcal{M}_{ \pm}$new coordinates by

$$
\begin{array}{ll}
q_{k}^{+}=\frac{1}{\sqrt{2}}\left(q_{k}-q_{2 r+2-k}\right), \quad p_{k}^{+}=\frac{1}{\sqrt{2}}\left(p_{k}-p_{2 r+2-k}\right), & k=1, \ldots, r ; \\
q_{k}^{-}=\frac{1}{\sqrt{2}}\left(q_{k}+q_{2 r+2-k}\right), \quad p_{k}^{-}=\frac{1}{\sqrt{2}}\left(p_{k}+p_{2 r+2-k}\right), \quad k=1, \ldots, r ; \\
q_{r+1}^{-}=q_{r+1}, \quad p_{r+1}^{-}=p_{r+1},
\end{array}
$$



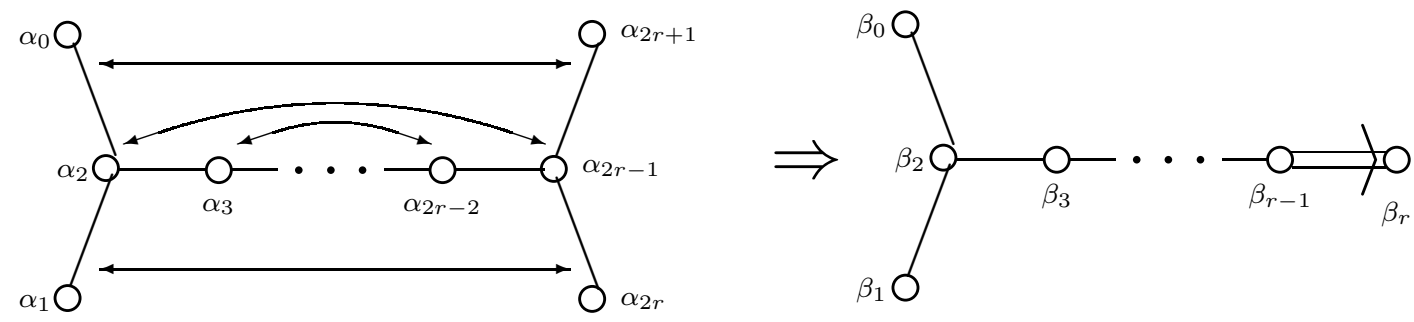

Figure 7. Reductions of $\boldsymbol{D}_{2 r+1}^{(1)}$ to $\boldsymbol{B}_{r}^{(1)}$.

i.e. $\operatorname{dim} \mathcal{M}_{+}=2 r$ and $\operatorname{dim} \mathcal{M}_{-}=2 r+2$.

This involution induces an involution $\mathcal{C}^{\#}$ of the Kac-Moody algebra $\boldsymbol{D}_{2 r+1}^{(1)}$ which acts on the root space as follows (see fig. (7):

$$
\begin{array}{r}
\mathcal{C}^{\#} e_{k}=-e_{2 r+2-k}, \quad k=1, \ldots, 2 r+1 ; \\
\mathcal{C}^{\#} \alpha_{k}=\alpha_{2 r+1-k}, \quad k=0, \ldots, 2 r .
\end{array}
$$

The involution $\mathcal{C}^{\#}$ splits the root space $\mathbb{E}^{2 r+1}$ into a direct sum of its eigensubspaces: $\mathbb{E}^{2 r+1}=\mathbb{E}_{+} \oplus \mathbb{E}_{-}$with $\operatorname{dim} \mathbb{E}_{+}=r$ and $\operatorname{dim} \mathbb{E}_{-}=r+1$. The restriction of $\pi$ onto $\mathbb{E}_{+}$ leads to the admissible root system $\pi^{\prime}=\left\{\beta_{0}, \ldots, \beta_{r}\right\}$ of $\boldsymbol{B}_{r}^{(1)}$ :

$$
\beta_{k}=\frac{1}{2}\left(\alpha_{k}+\mathcal{C}^{\#} \alpha_{k}\right), \quad k=0, \ldots, r
$$

The subspace $\mathbb{E}_{-}$is spanned by the vectors $\gamma_{k}=\frac{1}{2}\left(\alpha_{k}-\mathcal{C}^{\#} \alpha_{k}\right), k=1, \ldots, r+1$. The RHF is described by the following densities $\mathcal{H}_{7}^{\mathbb{R}}, \omega_{7}^{\mathbb{R}}$ :

$$
\begin{aligned}
& \mathcal{H}_{7}^{\mathbb{R}}=\frac{1}{2} \sum_{k=1}^{r} p_{k}^{+2}-\frac{1}{2} \sum_{k=1}^{r+1} p_{k}^{-2}+\sum_{k=2}^{r} 4 e^{-\left(\boldsymbol{q}_{+}, \beta_{k}\right)} \cos \left(\boldsymbol{q}_{-}, \gamma_{k}\right) \\
& +2 e^{-\left(\boldsymbol{q}_{+}, \beta_{0}\right)} \cos \left(\boldsymbol{q}_{-}, \gamma_{0}\right)+2 e^{-\left(\boldsymbol{q}_{+}, \beta_{1}\right)} \cos \left(\boldsymbol{q}_{-}, \gamma_{1}\right), \\
& \omega_{7}^{\mathbb{R}}=\sum_{k=1}^{r} \delta p_{k}^{+} \wedge \delta q_{k}^{+}-\sum_{k=1}^{r+1} \delta p_{k}^{-} \wedge \delta q_{k}^{-} .
\end{aligned}
$$

\section{Conclusions}

We outlined the generalization of the notion of RHF to infinite dimensional Hamiltonian systems using as a basis the ATFT type models. We established that the special properties of these models allow us to relate the construction of the RHF to the study of $\mathbb{Z}_{2}$ symmetries of the extended Dynkin diagrams. The general construction is illustrated by several examples of such models related to the type $\mathbf{A}_{n}$ and $\mathbf{D}_{n}$ Kac-Moody algebras.

The ATFT models obtained here are generalizations of the ATFT in [6]. Indeed, they contain two types of fields $\boldsymbol{q}_{+}(x, t)$ and $\boldsymbol{q}_{-}(x, t)$ which have different properties with 
respect to the involution $\mathcal{C}$, namely $\mathcal{C} \boldsymbol{q}_{ \pm}(x, t)= \pm q_{ \pm}(x, t)$. The $\mathbb{Z}_{2}$-reductions analyzed in [6] contain only one type of fields invariant with respect to $\mathcal{C}$.

Some additional problems are natural extensions to the results presented here. The first one is the complete classification of all RHF of ATFT, including the cases related to the exceptional Kac-Moody algebras. Next is the derivation of their Hamiltonian properties based on the classical $R$-matrix approach, see [14. The last and technically more involved problem is to solve the inverse scattering problem for the Lax operator and thus prove the complete integrability of all these models.

In [15] it was shown that the dynamics of different RHF for the finite-dimensional Toda systems may be qualitatively different; for example some of the non-compact trajectories may become compact. Similar qualitative changes may be expected also in the infinitedimensional cases.

Another interesting problem is related to the fact that the integrable systems possess a hierarchy of Hamiltonian structures, for review of the infinite-dimensional cases see e.g. [16, 17] and the references therein; for the (finite-dimensional) Toda chains see [18, 19. It is an open problem to construct the RHF for ATFT using some of its higher Hamiltonian structures.

Finally, this method provides a tool for systematic construction and classification of the RHF of a wide class of finite and infinite-dimensional Hamiltonian systems, which need not be necessarily integrable. Therefore to each Hamiltonian system one can associate a family of its RHF. To do this one has to construct the corresponding involutions of the Poisson brackets preserving the Hamiltonian.

Acknowledgments. One of us $(G G G)$ thanks the organizing committee of the European Research Conference on "Symmetries and Integrability of Difference Equations" (SIDE VI) for the scholarship and for the warm hospitality in Helsinki and the Expert Council for Young Scientists of the Bulgarian Academy of Sciences for financial support. We also acknowledge support by the National Science Foundation of Bulgaria, contract No. F-1410.

\section{References}

[1] Gerdjikov V S, Kyuldjiev A, Marmo G and Vilasi G, Complexifications and Real Forms of Hamiltonian Structures, European J. Phys. 29B (2002), 177-182; Real Hamiltonian forms of Hamiltonian systems, European Phys. J. B. 38 (2004) 635-649.

[2] Mikhailov A V, The reduction problem and the inverse scattering problem, Physica D 3 (1981), 73-117.

[3] Gerdjikov V S, $Z_{N}$-reductions and new integrable versions of derivative nonlinear Schrödinger equations, in Nonlinear evolution equations: integrability and spectral methods, Editors: Fordy A P, Degasperis A, Lakshmanan M, Manchester University Press, 1981, 367-379.

[4] Gerdjikov V S, Grahovski G G, Ivanov R I and Kostov N A, N-wave interactions related to simple Lie algebras. $\mathbb{Z}_{2}$ - reductions and soliton solutions, Inv. Problems 17 (2001), 999-1015.

[5] Gerdjikov V S, Analytic and Algebraic Aspects of Toda Field Theories and their Real Hamiltonian Forms, in Bilinear Integrable Systems: from Classical to Quantum, Continuous to Discrete, Editors: Faddeev F, van Moerbeke P and Lambert F, NATO Science Series II: Mathematics, Physics and Chemistry 201, Springer, Dordrecht, 2005, in press. 
[6] Khastgir S P and Sasaki R, Instability of solitons in imaginary coupling affine Toda field theory, Prog. Theor. Phys. 95 (1996), 485-501; Non-canonical folding of Dynkin diagrams and reduction of affine Toda theories, Prog. Theor. Phys. 95 (1996), 503-518.

[7] Gerdjikov V S and Yanovski A B, Completeness of the eigenfunctions for the Caudrey-BealsCoifman system, J. Math. Phys. 35 (1994), 3687-3725.

[8] Humphreys J E, Reflection Groups and Coxeter Groups, Cambridge University Press, Cambridge, 1992.

[9] Mikhailov A V, Olshanetzky, M A, Perelomov, A M, Two-dimensional generalized Toda lattice, Commun. Math. Phys. 79 (1981), 473-490.

[10] Olive D, Turok N and Underwood J W R, Solitons and the energy-momentum tensor for affine Toda theory, Nucl. Phys. B401 (1993), 663; Affine Toda Solitons and Vertex Operators, Nucl. Phys. B409 (1993), 509.

[11] Evans J, Complex Toda theories and twisted reality conditions. Nucl. Phys. B 390 (1993), 225

Evans, J and Madsen J O, Nucl. Phys. B 536 (1999), 657-703; Erratum-ibid. 547 (1999) 665; Evans J and Madsen J, Real form of non-Abelian Toda theories and their $W$-algebras. Phys. Lett. B 384 (1996), 131.

[12] Helgasson S, Differential geometry, Lie groups and symmetric spaces, Academic Press, 1978.

[13] Kac V G, Infinite Dimensional Lie Algebras, Cambridge University Press, 1990.

[14] Sklyanin E K, Quantum version of the method of Inverse scattering problem. Sci. Notes. LOMI seminars 95 (1980), 55-128 (In Russian), Translated in J. Sov. Math. 19, No. 5 (1982), 1546 - 1596; Kulish, P P Quantum difference nonlinear Schrodinger equation. Lett. Math. Phys. 5 (1981), 191-197.

[15] Gerdjikov V S, Evstatiev E G and Ivanov R I, The complex Toda chains and the simple Lie algebras -solutions and large time asymptotics. J. Phys. A 31 (1998), 8221-8232; The complex Toda Chains and the Simple Lie Algebras - Solutions and Large Time Asymptotics - II. J. Phys. A 33 (2000), 975-1006.

[16] Drinfel'd V and Sokolov V V, Lie Algebras and equations of Korteweg - de Vries type. Sov. J. Math. 30 (1985), 1975-2036.

[17] Gerdjikov V S, Algebraic and Analytic Aspects of $N$-wave Type Equations. Contemporary Mathematics 301 (2002), 35-68.

[18] Damianou P A, Multiple Hamiltonian Structure of the Bogoyavlensky-Toda Lattices, Rev. Math. Phys. 16 (2004), 175-241.

[19] Damianou P A, Multiple Hamiltonian Structure for Toda-type systems, J. Math. Phys. 35 (1994), 5511-5541. 